\title{
Automatic Documentation of Java Programs
}

\section{Dujan B. Taha}

Asmaa H. Thanoon

\section{dujan_taha@uomosul.edu.iq}

College of Computer Sciences and Mathematics

University of Mosul

Received on: 22/09/2013

Accepted on: 12/02/2014

\begin{abstract}
Documentation process plays a great role in software systems development and maintenance, and regarded as an important feature for all software projects and programs in general. In the past, documents had been generated manually by documents writers, but these documents were very weak and had many errors, Therefore, automated documentation had been invented due to its good features that is complete, precise, and cheap compared with manual ways.

The work concerns with constructing an automated tool ADT(Auto Documentation Tool) used for documenting source code for programs written in java programming languages in legacy systems particularly and for programs in general which does not have any documentation at all or it were documented poorly in order to make them, and producing as a result of the document process either the class diagrams using UML (Unified Modeling Language) or producing easy understandable textual output using XML (Extensible Markup Language).
\end{abstract}

Keywords: Automatic Documentation, UML, XML.

$$
\text { التوثيق الالي لبرامج جافا }
$$

$$
\begin{aligned}
& \text { اسماء هادي ذنون } \\
& \text { لدجان بشير طه } \\
& \text { كلية علوم الحاسوب والرياضيات } \\
& \text { جامعة الدوصل }
\end{aligned}
$$

201412112 : تاريخ قبول البحث

201319122 : تاريخ استلام البحث

\section{الملخص}

تؤدي عمليات التوثيق دورا كبيرا في تطوير الأنظمة البرمجية وصيانتها، إذ تعد هذه العمليات سمة مهمة

لكل مشاريع البرمجيات والبرامج على نحو عام. في البداية كانت الفكرة توليد الوثائق على نحو يدوي من قبل كتاب الوثائق في المجال الخاص بهم وذلك لمعرفتهم بكافة جوانب النظام ولتوليد وثائق أكثر وضوحا، ولكن هذه الطرائق كانت ضعيفة وعرضة للأخطاء, لذلك نشأت محاولات للقيام بعمليات التوثيق على نحو آلي لما يمتاز به التوثيق الالي من مزايا جيدة إذ تكون عمليات متكاملة، دقيقة، وغير مكلفة مقارنة بالطرق اليدويـة.

يتعلق البحث بيناء أداة توثيق آلية (ADT(Auto Documentation Tool) تستخدم في توثيق شفرة المصدر للبرامج المكتوبة بلغة جافا في الأنظمة القديمة (Legacy systems) ولاسيما البرامج على نحو عام والتي 
لا تحتوي على توثيق أو إعادة توثيق هذه الأنظمة بثكل متقن, وقد تم تمثيل ناتج عمليات التوثيق هذه إما على شكل مخطط الصنف (Class diagram) باستخدام لغة النمذجة الموحدة (Unified Modeling Language)

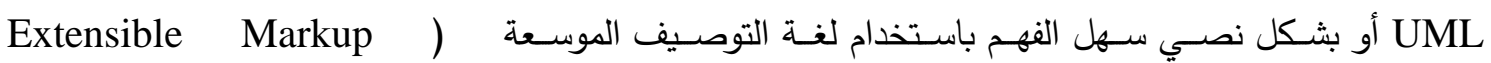
.XML(Language الكلمات المفتاحية: التوثيق الالي، XML،UML.

1. 1المقدمة

إن لعمليات توثيق البرمجيات بمساعدة الحاسوب دوراً فاعلاً في تطوير البرمجيات وهندستها، إذ أنها تساعد

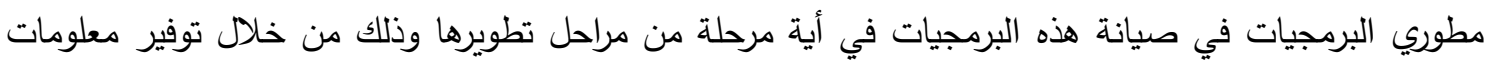

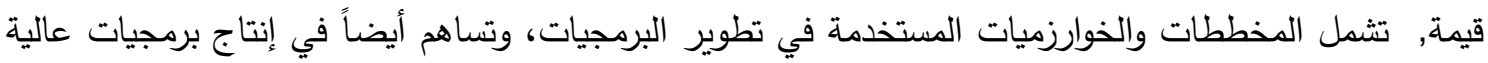
الجودة من خلال تقليل الأخطاء في كل مرحلة من مراحل تطوير هذه البرمجيات.

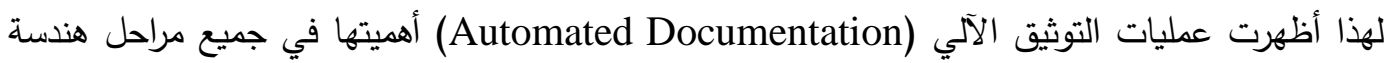
البرمجيات وتم الاستفادة من التوثيق الخاص بهذه البرمجيات من قبل مطوري البرمجيات، وبذلك تكون مهمة توثيق هيق هريق البرمجيات مهمة ليست سهلة ولاسيما عند توثيق الثفرة المصدرية (Source Code). أن مطوري البرمجيات يواجهون مهمة صعبة في توثيقها، ويقضون اغلب وقتهم في محاولة فهم الثفرة المصدرية، علاوة على ذلك فان

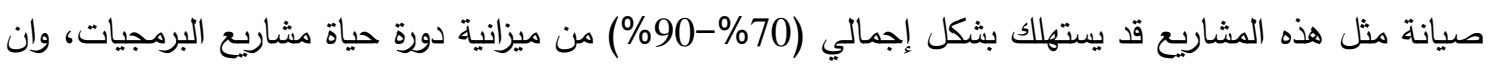
الحفاظ على جودة التوثيق العالية وتطويرها أمر حيوي لمساعدة المطورين في فهم الثفرة المصدرية وتعديلها

سيتم التطرق خلال البحث إلى مفهوم الهندسة العكسية والتي يُعرفها المختصون في مجال هندسة البرمجيات بأنها العملية التي تأخذ شفرة المصدر كإدخال وتعمل على تحويلها إلى مخطط رسومي يمثل شفرة المصدر في مرحلة التصميم، حيث تعمل الهندسة العكسية على إعادة بناء التقنيات أو المخططات (Charts) الدلالية المفقودة القواعد البيانات لمراحل تطور مشروع البرمجيات مما يتيح الاحتفاظ بإرث (Legacy) قواعد البيانات بثكل آمن وإن

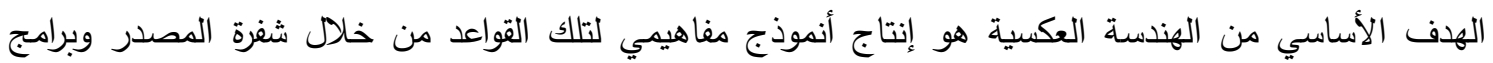
التطبيق [8]. تتاولت العديد من البحوث عمليات توثيق البرامج, وهنا سيتم عرض بعض البحوث المختلفة ذات العلاقة بموضوع البحث حيث قام الباحثان ويمر وبيوز [3] في عام 2008 بتطوير أداة آلية بشكل كامل \% وهذه الأداة ساكنة اذ وجد أن التوثيق الناتج من هذه الأداة يكون جيد بنسبة (Fully Automated Tool)

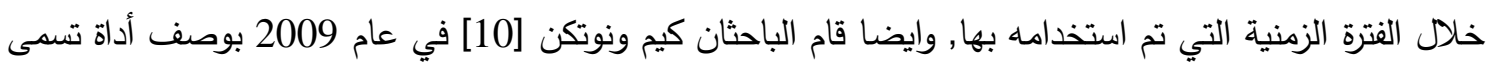
LSDiff (Systematic Structural Differences) هيكلية الثفرة المصدرية ولكن هنا عملية توثيق الثفرة تكون اكثر تفصيلا, وقامت الباحثة بارون [2] في عام 2010 بدراسة عمليات التوثيق على شفرة المصدر ومن خلال بحثها استطاعت تسهيل الأمر للمستخدمين في كيفية

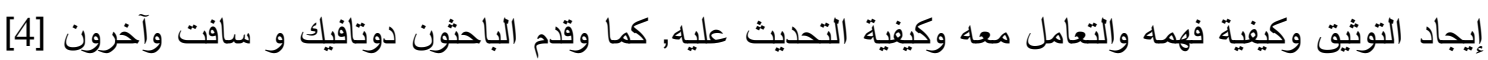


في عام 2011 أداة تعتمد في عملها على العمليات التي تتم على البرمجيات ليتم تحديد عيوب التوثيق وهذا يسهل

تحديد جودة عمليات التوثيق.

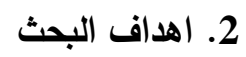

يهذف البحث إلى أن تكون أداة التوثيق المقترحة بالمواصفات الآتية: 1- تقوم بتوثيق الثفرة المصدرية بشكل آلي.

2- تحقيق واحدة من مفاهيم الهندسة العكسية وهي إعادة توثيق البرمجيات (Redocumentation)

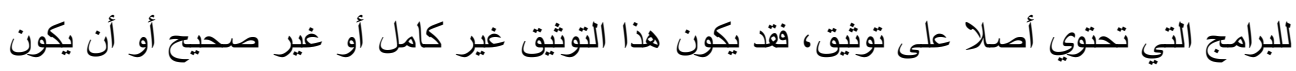

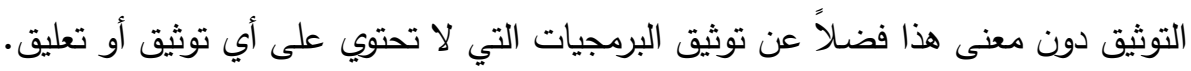

3- تحقيق أسلوب التوثيق المرئي من خلال استخدام مخططات لغة النمذجة الموحدة, فضلا عن تحقيق

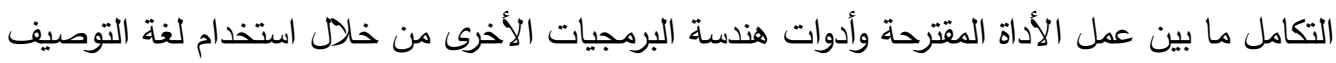

$$
\text { الموسعة في تمثيل البيانات الموثقة. }
$$

\section{3. اعادة هندسة البرمجيات والهندسة العكسية}

تعرف عمليات إعادة هندسة البرمجيات على أنها دراسة وتعديل أي نظام وإعادة تثكيله بصيغة جديدة أي هي إعادة هندسة وتعديل النظام وعموما تكون هذه العمليات لإغراض إضافة وظيفة جديدة أو لتصحيح الأخطاء.

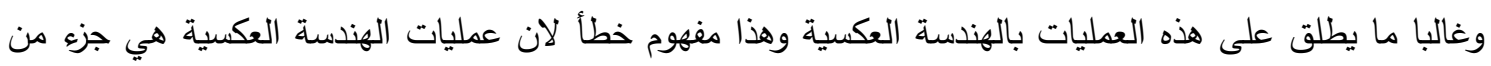

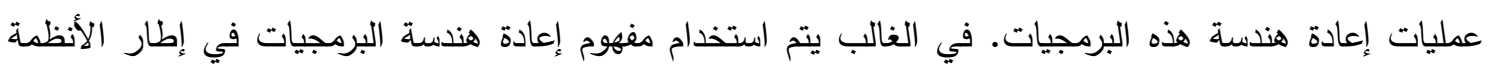

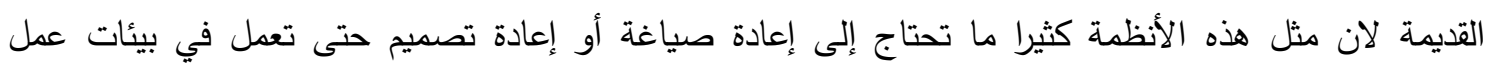
جديدة [13].

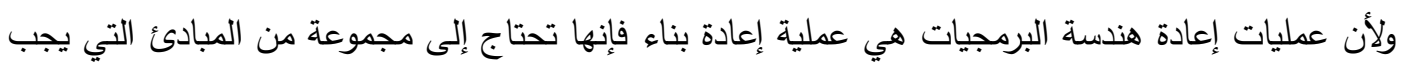
إتباعها, ومن هذه المبادئ تحديد متطلبات إعادة البناء وهل هذه المتطلبات من المدكن إعادة نمذجتها, وبعد البداء

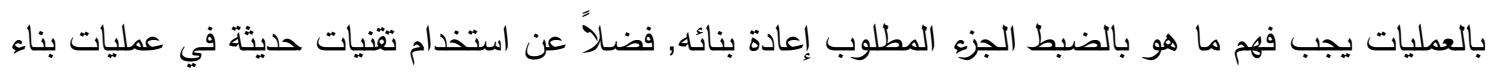
النظام الجديد حتى ولو كانت مكلفة ولكن ذلك سوف يجنبنا إضاعة أو استهلاك الوقت خلال مرحلة الصيانة وأخيرا تحقيق الجودة في المنتج أو النظام الناتج من خلال عمليات البناء هذه. ولتتفيذ هذه المبادئ يجب استخدام نموذج إنهات عمليات إعادة هندسة البرمجيات (Software Reengineering process model) الذي يتضمن ست فعادئ فئيات كما موضح بالثكل (1) إذ انه في بعض الأحيان يتم تتفيذ هذه العمليات على نحو متسلسل ولكن هذا لا يحدث

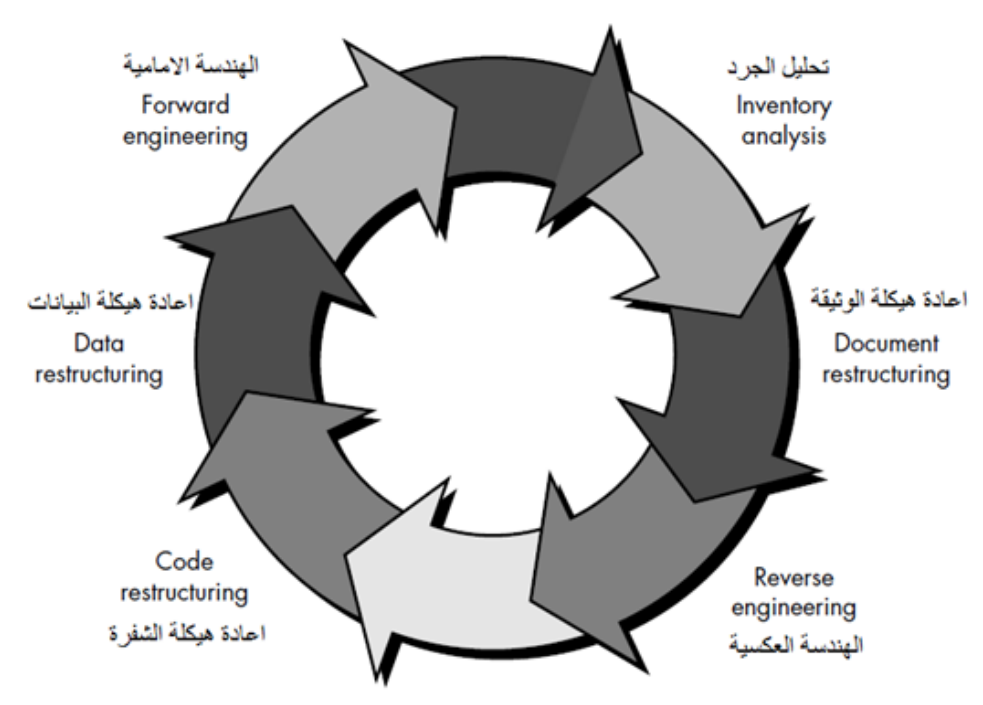




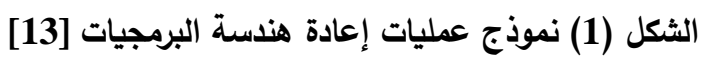

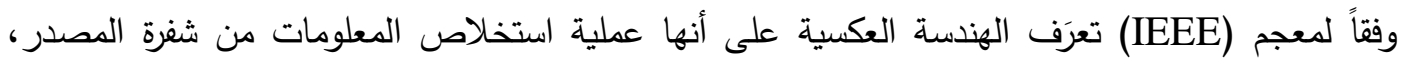
تعرَف أيضا على أنها ذلك الجزء من البرمجيات الذي يتمثل في استعادة أو إعادة البناء الوظيفي لمواصفاتها التقنية [1]، ويتم ذلك من خلال عملية التعرف على مكونات النظام والعلاقات التي تربط تلك المكونات وتكوين تمثيل للنظام في شكل أخر، كما تعد الهندسة العكسية من أهم فروع التصميم الهندسي وتصنيع التطبيقات خطوة

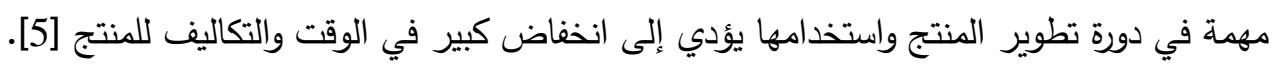

4. اسباب تنفيذ الهندسة العكسية

هنالك نوعان من المبررات الأساسية لتنفيذ الهندسة العكسية وكالاتي:

أولا: يتم تتفيذ الهندسة العكسية من أجل فهم كيف يمكن لبرنامج الحاسوب أن يعمل فعلاً.

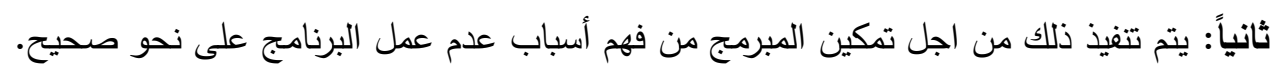

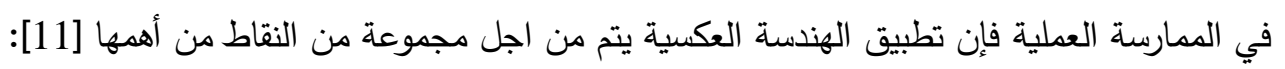

$$
\text { 1- وضع برنامج جديد يتعامل مع البرنامج الأصلي. }
$$

2- وضع برنامج جديد يتعامل مع بيانات البرنامج الأصلي.

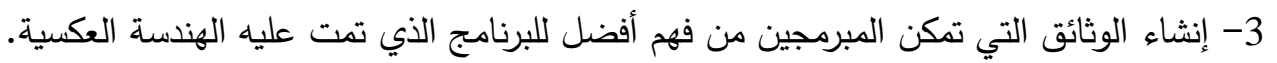

4- اختبار أمنيَة البرنامج والتحقيق في احتمال خرق الأمنيَّة.

\section{5. - 5واعي عملية التوثيق}

التوثيق مهم في كل مراحل دورة حياة البرمجيات كما هو معلوم ولاسيما مرحلة توثيق شفرة المصدر , حيث يوفر اتصالاً أفضل مابين المتطلبات, يفيد في استخدام التصاميم القديمة, يسهل عملية التكامل ما بين وحدات المشروع المكتوبة على نحو منفصل, وأيضا يفيد في مراجعة التصاميم, كما يزيد من فعالية استخدام الثفرة

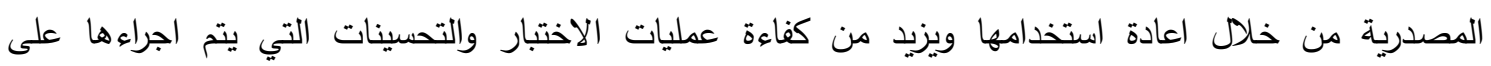
المنتج [12]. فضلاً عن انه يجعل أعضاء الفريق في تواصل مستمر , يعمل كمستودع معلومات سيستفيد منه مهندسو الصيانة, يوفر معلومات كافية تسمح لأعضاء ادارة المشروع بإدارة كافة النشاطات كالميزانية والخطط وجدولة عمليات المشروع ويوصف التوثيق للمستخدم كيف يتم تثغيل النظام وإدارته. وأخيرا يجب أن تستمر عملية التوثيق حتى بعد كتابة الثفرة [14,9] •

\section{6. الخوارزمية العامة للأداة المقترحة}

تمر الأداة المقترحة ADT بعدد من الرراحل والتي تم توضيحها من خلال الثكل (2). إذ تبدأ عملية

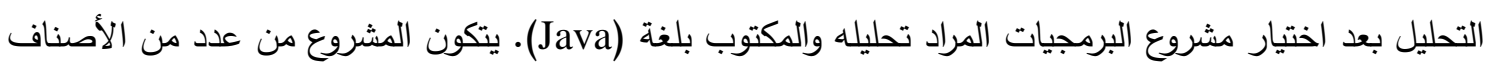

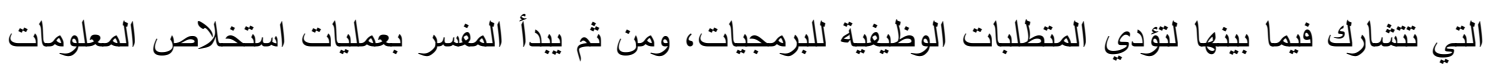

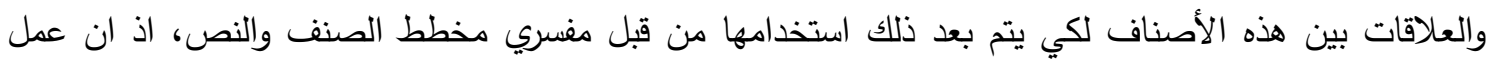
المفران بثكل متواز يكون من خلال ان المعلومات المستحصلة من تحليل كل صنف بلان بالاضافة الى العلاقات مابين هذه الاصناف يتم استخدامها في نفس الوقت في عملية التوثيق بثكل رسومي مرئي باستخدام مخطط 
الصنف وايضا التوثيق بثكل نصي على شكل تقارير XML وهذا يفيد في تقليل الوقت إذ أن كليهما يعتمد على نفس المعلومات والعلاقات التي تم استخلاصها من المشروع البرمجي المراد تحليله لغرض توليد الوثائق.

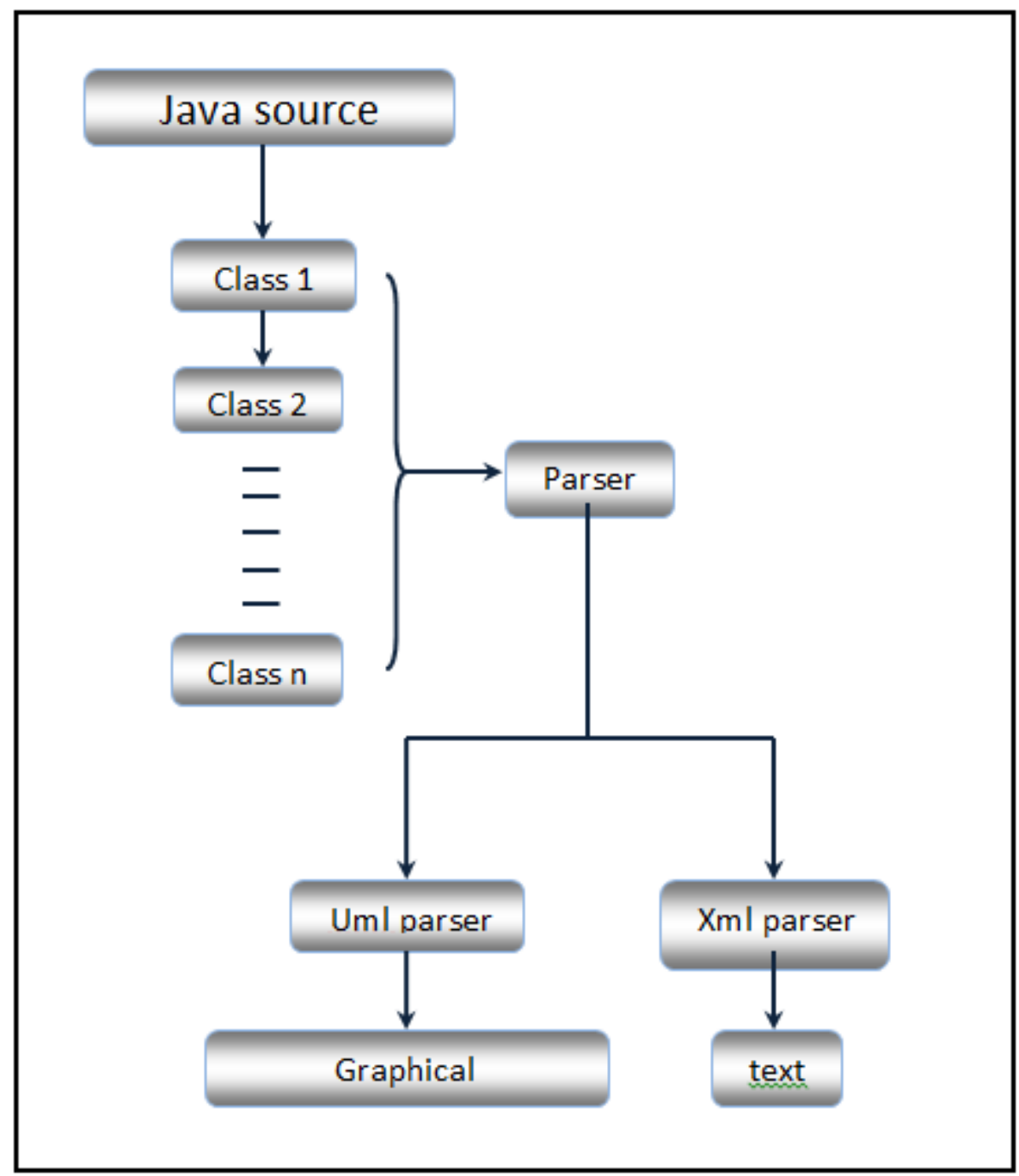

الثكل (2) المخطط العام للأداة المقترحة

UML 1.6. خوارزمية رسم مخطط

تم تمثيل نتائج تتفيذ هذه الخوارزمية من خـلال استخدام احد مخططات لغة UML لان نـاتج التنفيذ هنا

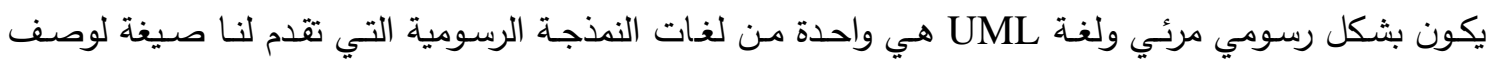

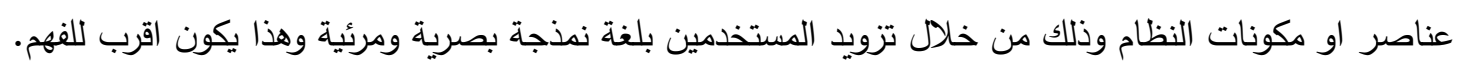

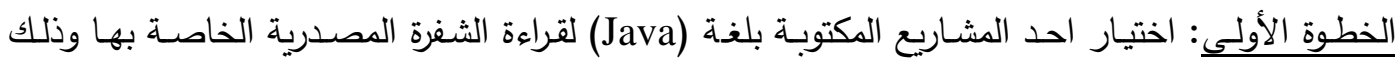

$$
\text { لغرض توليد توثيق آلي لها. }
$$

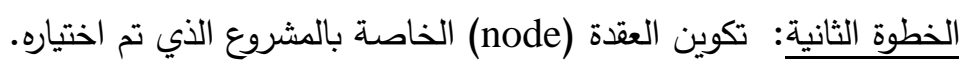

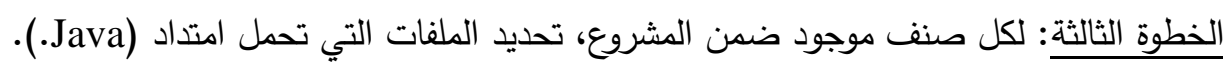
الخطوة الرابعة: استدعاء المحلل لتحليل محتويات الصنف وتحديد الأجزاء الأتية:

1 - تحديد اسم الحزمة.

2- تحديد اسم الصنف. 
3- تحديد الصنف الذي يمثل علاقة الوراثة. 4- تحديد الصنف الذي يمثل علاقة تمثيل الواجهات. 5- تحديد الدوال فضلا عن دوال البناء.

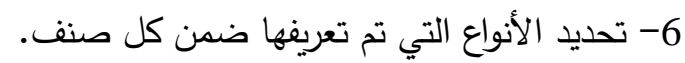
وبعد تحديد الأجزاء أعلاه ضمن الصنف الواحد يتم استدعاء بقية الأصناف تباعا إلى حين انتهاء جميع الأصناف المكونة للمشروع المراد توثيقه، وبعدها يتم خزن المعلومات المحددة.

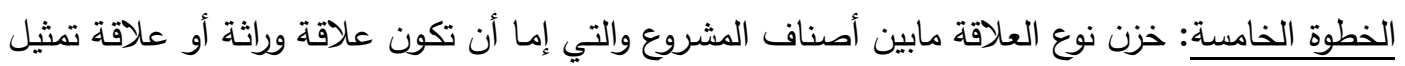
الواجهات وأحيانا الاثثين معا.

الخطوة السادسة: رسم العقدة الخاصـة بكل صنف ضمن مخطط الصنف مع توضيح محتويات كل صنف وما يحتويه من أجزاء.

الخطوة السابعة: تمثيل العلاقات مابين العقد الموجودة ضمن مخطط الصنف والتي تمثل الأصناف المكونة للمشروع وتوضيح هذه العلاقة بالرسم الذي يظهر على واجهة المستخدم الرسومية.

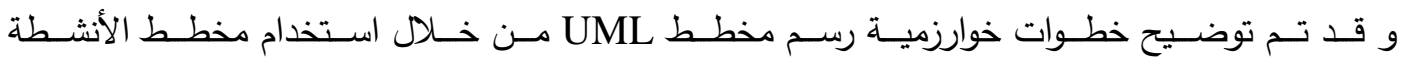

(UML activity diagram)

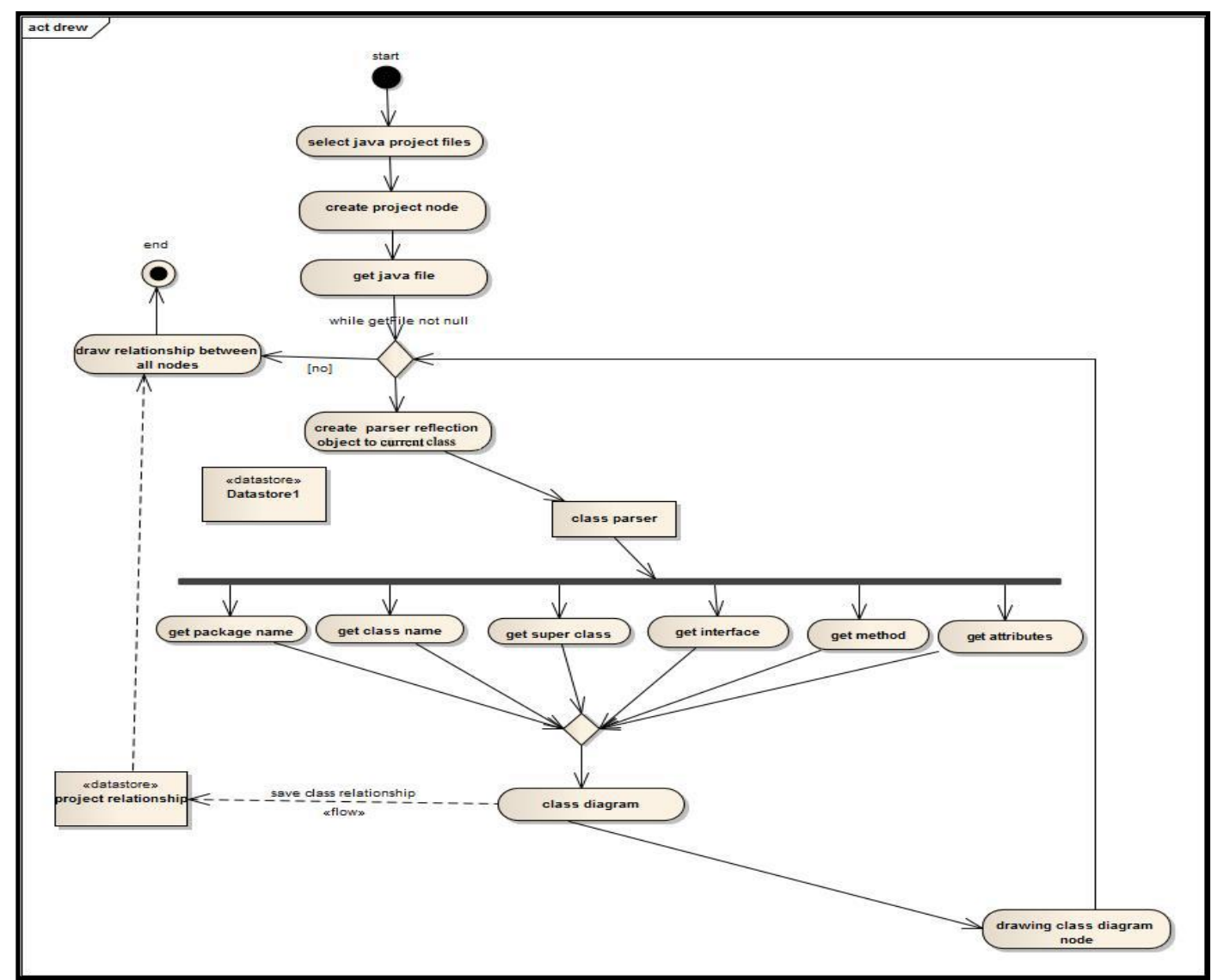

الثكل (3) مخطط الأنشطة لخوارزمية رسم مخطط

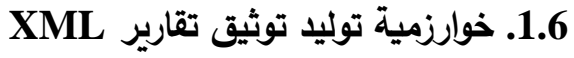

الخطوة الأولى:_اختيار احد المشاريع المكتوبة بلغة (Java) لقراءة الثفرة المصدرية الخاصة بها. 
الخطوة الثانية: تكوين العقدة الخاصة بالمشروع الذي تم اختياره. الخطوة الثالثة: لكل صنف موجود ضمن المشروع، تحديد الملفات التي تحمل امتداد (Java.). الخطوة الرابعة: استدعاء المحلل وإتباع مايأتي:

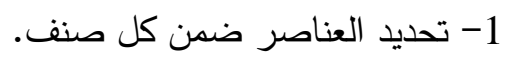
2- إيجاد العلاقات ما بين الأصناف.

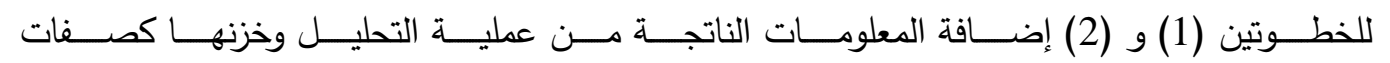
في ملف Attributes)

3- لكل صنف ضمن المشروع تحديد جميع الدوال الموجودة في الصنف الواحد وإضافتها كعناصر إلى ملف XML .XML

4- تحديد المتغيرات داخل كل صنف وإضافتها إلى ملف XML. الخطوة الخامسـة: تعاد الخطوات (1)، (2)، (3) و (4) إلى حين النتهاء تحليل جميع الأصناف الككونـة للمشروع. وقد تـم توضـيح خطوات خوارزميـة توليــ تقارير XML من خـلال إستخدام مخطط الأنشطة الموضـح

بالثكل (4).

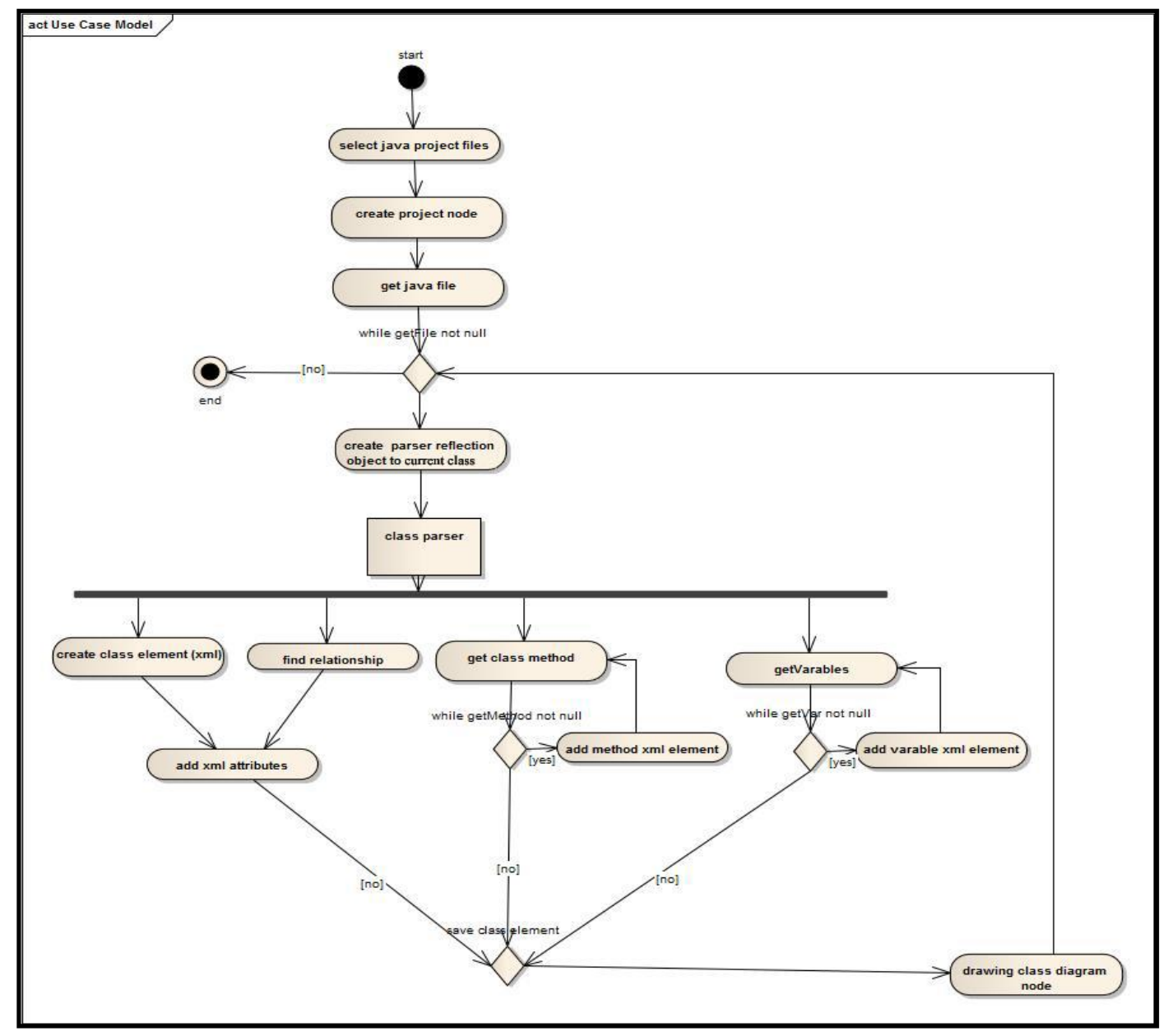

XML الثكل (4) مخطط الأنشطة لخوارزمية توليد تقرير 
تم تطبيق الأداة المقترحة على أكثر من نظام برمجي وذلك لتوضيح قدرة النظام على تمثيل علاقات الوراثة

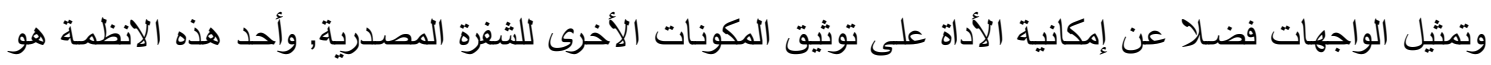

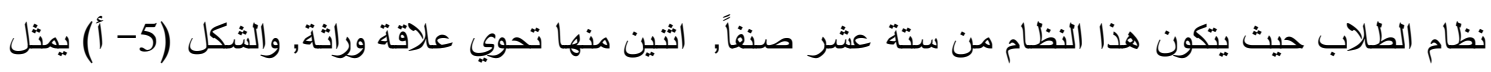
مخطط الصنف الخاص بهذا النظام ككل, وقد تم تحديد الصنف (Semester) بالثكل الدائري لعرض مكوناته بشكل تفصيلي من خلال الثكل (5- ب) اما علاقة الوراثة فقد تم تحديدها بالثكل المستطيل وكما موضح بالثكل 


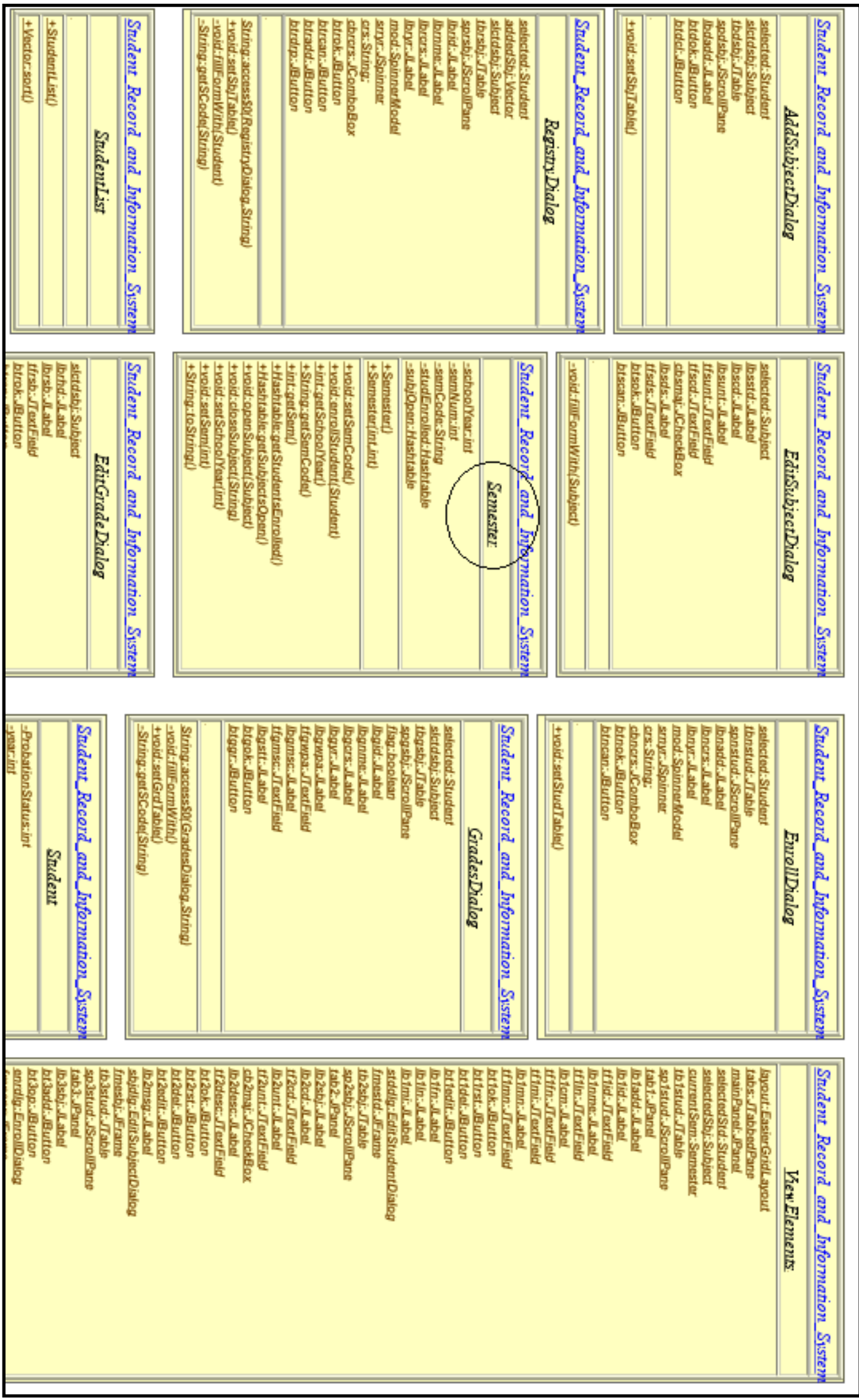

الثكل (5- أ) مخطط الصنف الخاص بتمثيل نظام الطلاب 


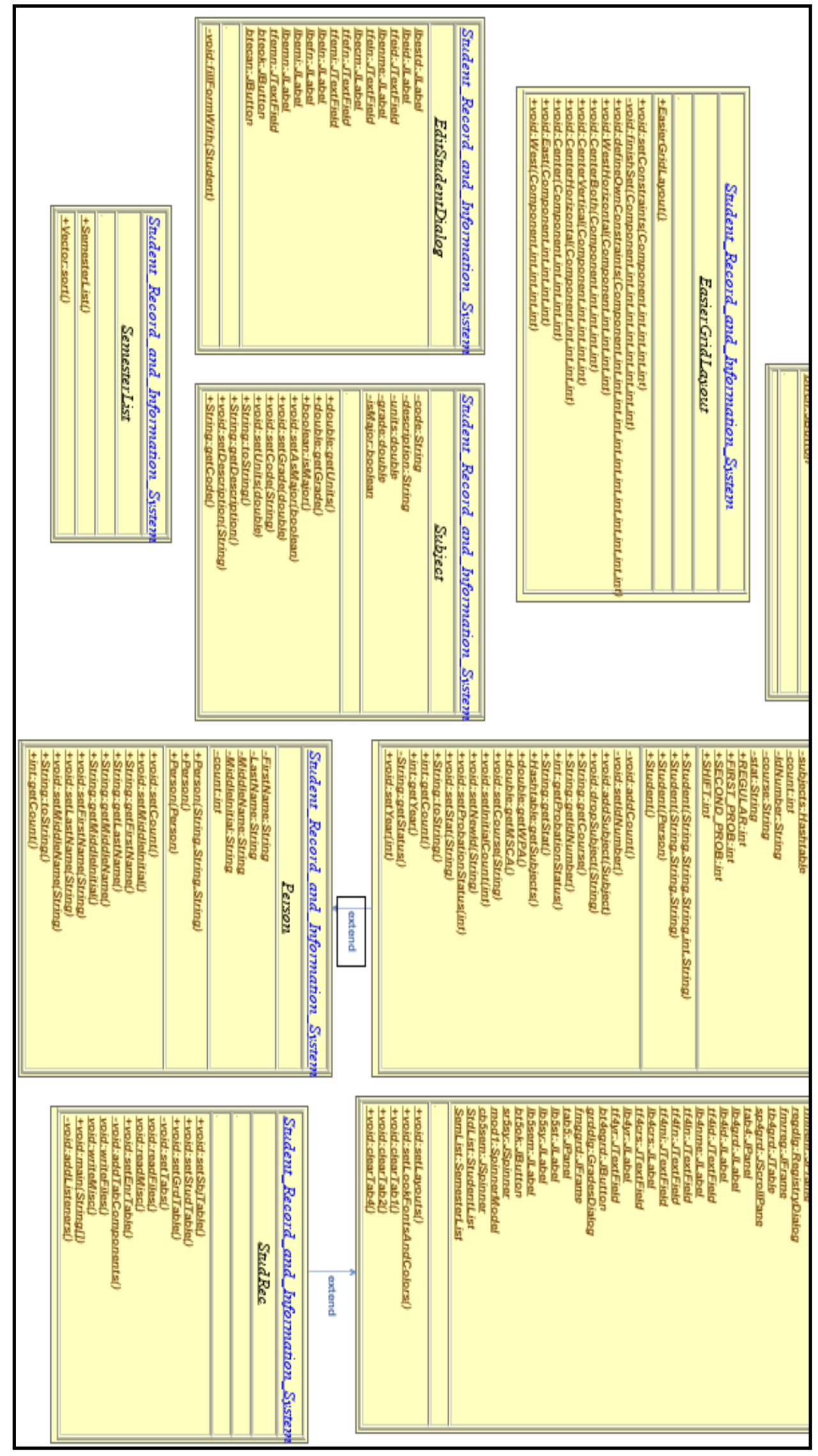

الثكل (5- أ) مخطط الصنف الخاص بتمثيل نظام الطلاب(تكملة) 


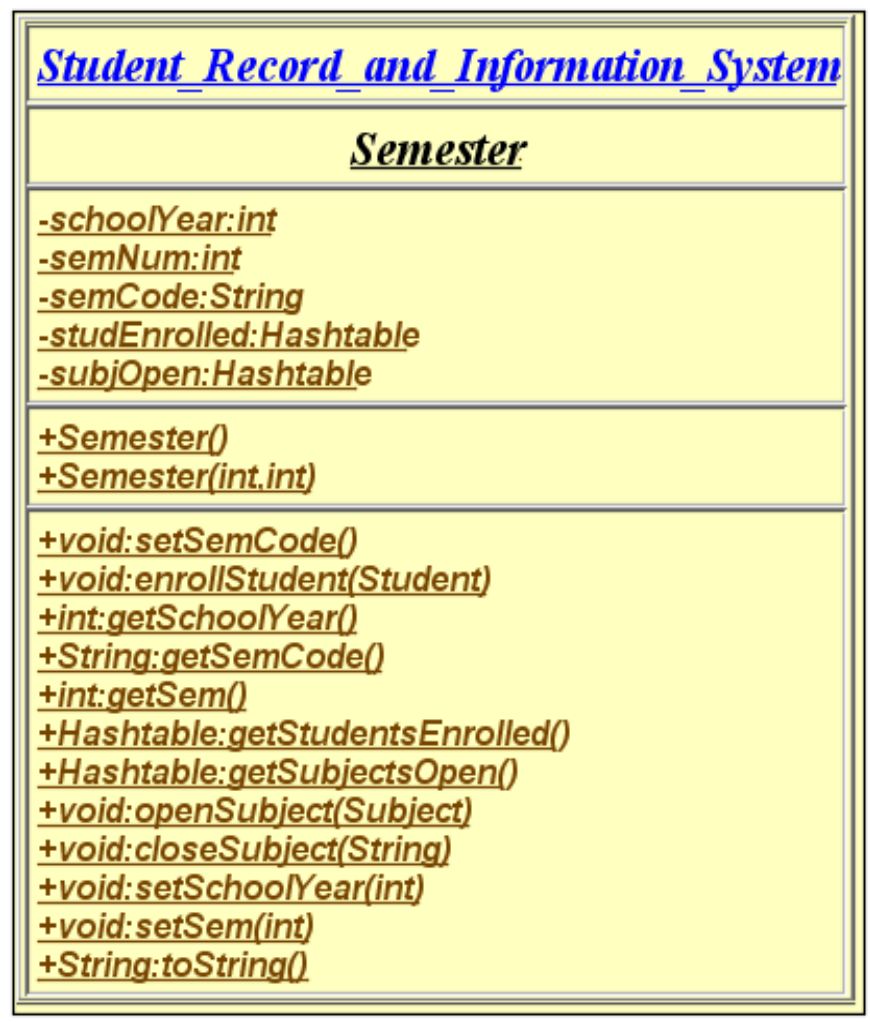

الثكل (5- ب) مخطط الصنف الخاص بتمثيل الصنف (Semester)

بعد تطبيق الاداة ADT على نظام الطلاب نلاحظ انه تم توثيق كافة الاصناف المكونة للنظام بضدنها

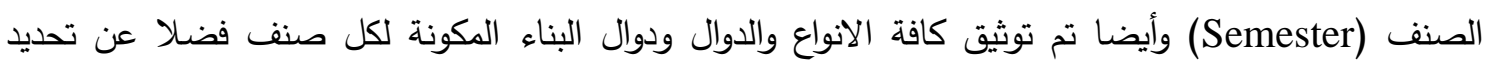
وتوثيق علاقة الوراثة وهذا موضح بالثكل (5- أ) و (5- ب), وعملية التوثيق هنا كانت بثكل رسومي مرئي.

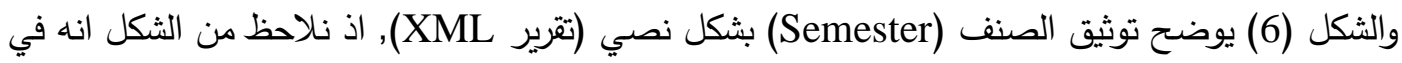
البداية تم تحديد اسم الصنف ومن ثم تحديد توثيق علاقة الوراثة من خلال تحديد اسم الصنف المتوارث, وبعدها تحديد اسم الصنف الذي يمثل علاقة الواجهات في حال وجودها, فضلا عن تحديد الانواع المعرفة وبعد ذلك تم تحديد دوال البناء ومن تعريف الدوال من خلال تحديد اسمها ونوعها هل هي عامة ام خاصة ام محمية وتحديد نوع واسم المتغيرات المستلمة مع تحديد نوع الارجاع ومن ثم انهاء الصنف بعد تحديد مكوناته, وبنفس الطريقة يتم توثيق الاصناف الاخرى الخاصة بالنظام. 


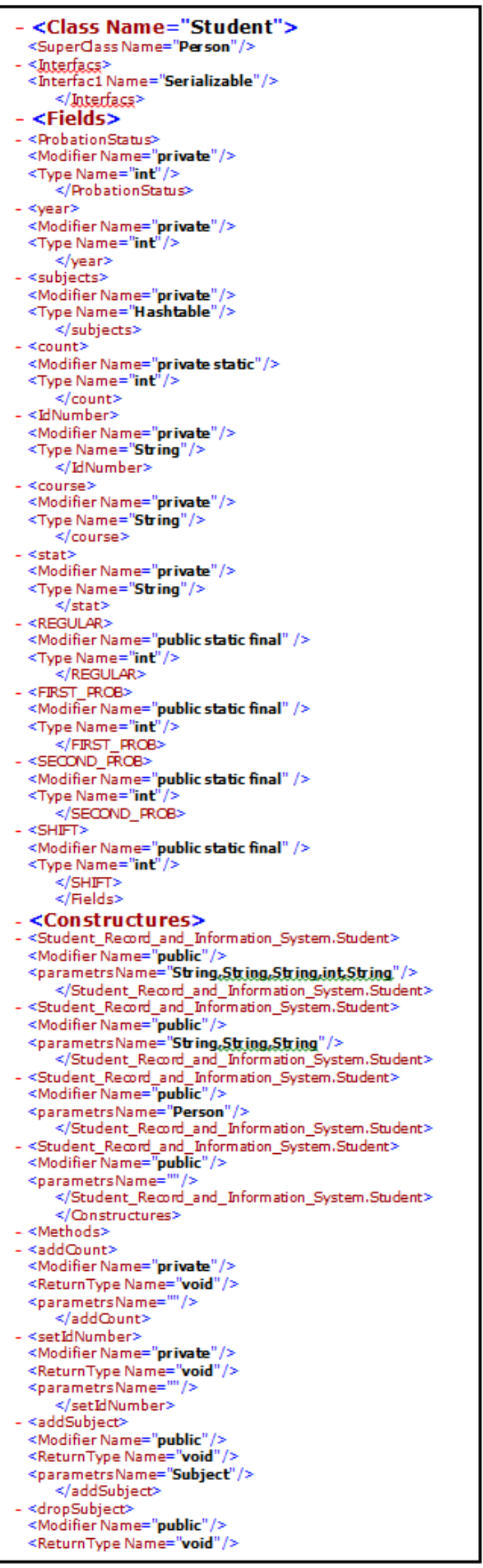

$</$ dropSubjects

<getCourse>

<Modifier Name="public" />

$<$ ReturnType Name="class java.lang.String" / >

$<$ parametrs Name $="+1 />$ $</$ getCourse

$<$ getIdNumber

$<$ Modifier Name="public" / >

$<$ ReturnType Name="class java.lang.String" / >

$<$ parametrs Name $=" '+1 />$

$</$ getId Numbers

$<$ getProbationStatus

<Modifier Name="public" />

$<$ ReturnType Name="int" $/>$

<parametrs Name="'" />

$</$ getProbationStatus $>$

$<$ getStat>

<Modifier Name="public" / >

$<$ ReturnType Name="class java.lang.String" / >

$<$ parametrs Name $=" 1 /$ /

$$
\begin{aligned}
& </ \text { getStat }> \\
& \text { etSubjects> }
\end{aligned}
$$

$<$ Modifier Name="public" / >

$<$ Return Type Name="class java.util.Hashtable" / >

$<$ parametrs Name $="$ "' /

$</$ getSubjects $>$

$<$ getWPA>

$<$ Modifier Name="public" />

$<$ ReturnType Name="double" / >

$<$ parametrs Name $="$ "' $/$ /

$</$ getWPA;

sgetMsos

$<$ Modifier Name="public" / >

$<$ ReturnType Name="double" / >

$<$ parametrs Name $="$ "'" $/>$

$$
</ \text { getMSOA> }
$$

$<$ Modifier Name="public"

$<$ ReturnType Name="void"/>

$<$ Return Type Name="void"/>
$<$ parametrs Name="Sting"/>

$</$ setCourse

$<$ setInitialCount

<Modifier Name="public static" / >

$<$ ReturnType Name="void" / >

<parametrs Name="int" /

$<$ /setInitialCount $>$

<setNewId>

$<$ Modifier Name="public" />

$<$ ReturnType Name="void" / >

$<$ ReturnType Name="void"/>
$<$ parametrs Name="String" /

$</$ setNewId $>$

<setProbationStatus?

<Modifier Name="public" />

$<$ ReturnType Name="void"/>

<parametrs Name="int" />

$</$ setProbationStatus $>$

$<$ set5tat>

$<$ Modifier Name="public" />

$<$ ReturnType Name="void" / >

$<$ parametrs Name="String" /> $</$ setStat $>$

$<$ Modifier Name="public" / >

$<$ ReturnType Name="class java.lang.String" / >

$<$ parametrs Name $="+1 /$ >

$</$ toString $>$

<getCount>

<Modifier Name="public static" / >

$<$ ReturnType Name="int" / >

$<$ parametrs Name $="+1 /$ >

$</$ getCount $>$

$$
\text { <getYear> }
$$

$<$ Modifier Name="public" / >

$<$ Return Type Name="int" / >

$<$ parametrs Name $={ }^{\prime \prime \prime} />$

$</$ getYear>

<getStatus

<Modifier Name="private" / >

$<$ ReturnType Name="class java.lang.String" / >

$<$ parametrs Name $="$ "' $/$ >

$</$ getStatus?

$<$ setYear

$<$ Modifier Name="public" />

$<$ ReturnType Name="void"/

$<$ parametrs Name="int" / >

$</$ setYear $>$

$</$ Methods $>$

$</$ dass $>$

الثكل (6) توثيق الصنف (Student) باستخدام لغة XML 
8. الاستنتاجات والاعمال المستقبلية

تم في هذا البحث تصميم وبناء اداة الية ADT لتوثيق البرامج وتميزت الاداة عن الاعمال السابقة بما يلي: • تم تطبيق الاداة على البرامج القديمة التي لا تحتوي على توثيق اصلا او الانظمة التي تحتوي على تصل

$$
\text { توثيق ولكنه غير متكامل. }
$$

الاداة لها القابلية على توليد توثيق في كل عملية تنفيذ للنظام على شفرة المصدر وبعد إجراء أي تحديث أو إضافة عليه فضلا عن ان استخدام الأداة المقترحة سوف يقلل من الجهد والوقت وكلئ وكلا العاملين مهم في مشاريع هندسة البرمجيات.

إثبات أهمية لغة التوصيفات الموسعة في بناء الوثائق من خلال توليد توثيق متكامل خالي من

$$
\text { التعارضات والتكرارات. }
$$

تتفيذ واحد من أهداف الهندسة العكسية من خلال تطبيق عمليات إعادة التوثيق على الثفرات

البرمجية والبيانات التي تتعامل معها هذه الثفرات، وذلك من خلال إتباع أسلوب منهجي ومنظم (أداة

توثيق كافة الأنواع المعرفة والدوال ودوال البناء وعلاقات الوراثة وتمثيل الواجهات بالإضافة إلى دقة

نتائج التوثيق من خلال مقارنتها مع شفرة المصدر الأصلية للمشروع المعني بعملية التحليل. التوثيق الناتج مقروء" (Readable) وواضحاً وهذا يسهل فهم الثفرة بشكل سريع وأيضا سهولة تتبع

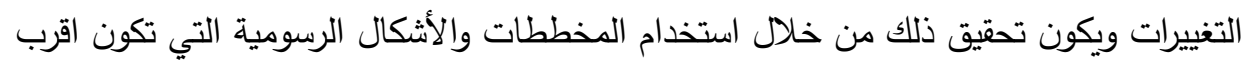
للفهج.

وفيما يخص الأداة المقترحة فان من أهم التوصيات المستقبلية لتطويرها هي عمل تكامل لإخراج الأداة المقترحة مع عمل أداة أخرى من ضمن أدوات هندسة البرمجيات, وايضا تتفيذ عمليات الهندسة العكسية لتحويل

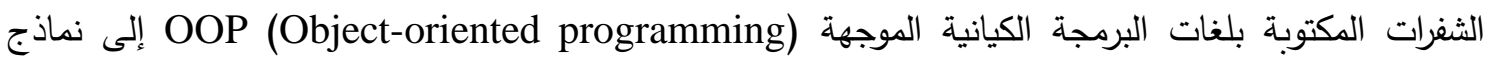
أخرى مكتوبة بلغة النمذجة الموحدة مثل مخطط الانشطة أو أي أنموذج آخر . 


\section{المصادر}

[1] Abraham Silberschatz, Henry F. Korth and S. Sudarshan, (2006), "Database System Concepts", McGraw-Hill.

[2] Baron, R., (2010), " Discrimination in the Documentation of Open Source Software ", Degree of Bachelor of Science, Professional Writing, Worcester Polytechnic Institute, USA.

[3] Buse, Raymond P.L., Weimer ,Westley R., (2008), " Automatic Documentation inference for exceptions", ISSTA '08 Proceedings of the 2008 international symposium on Software testing and analysis, PP: 273- 282, ISBN: 978-1-60558-050-0, doi>10.1145/1390630.1390664.

[4] Dautovic A., Plosch R. , Saft M., (2011), "Automatic Checking of Quality Best Practices in Software Development Documents", Quality Software (QSIC), 11th International Conference, IEEE Computer Society, PP: 208-217.

[5] Eyup Bagci, (2009), "Reverse engineering applications for recovery of broken or worn parts and re-manufacturing: Three case studies", Advances in Engineering Software(Elsevier), Vol.40 p.p 407-418.

[6] Friesen, A., Lemcke, J., Oberle, D., Rahmani, T., (2008), "Description of Functional and Non-Functional Requirements", SAP Research CEC, Karlsruhe, Germany, Project co-funded by the European Commission and the Swiss Federal Office for Education and Science within the Seventh Framework Programme, http://most-project.eu.

[7] Hallam P.,(Jan 2006), "What do programmers really do anyway?", In Microsoft Developer Network (MSDN) | C\# Compiler.

[8] Jean-Luc Hainaut, (2009), "Legacy and Future of Data Revers Engineering",16th Working Conference on Reverse Engineering, IEEE p.p.4-4.

[9] Kamthan, P., (2007), "On the Prospects and Concerns of Integrating Open Source Software Environment in Software Engineering Education", Concordia University, Montreal, Quebec, Canada, Journal of Information Technology Education, Volume 6.

[10] Kim M. and Notkin D., (2009), "Discovering and representing systematic Code changes", In International Conference on Software Engineering, pages 309-319.

[11] Leif Gamertsfelder, (2003), "Software reverse engineering - the current state of Australian law", Computer Law \& Security Report (Elsevier), Vol.19 no. 5 p.p 394-400.

[12] Parnas, D. L., (2011), "Precise Documentation: The Key to Better Software", The Future of Software Engineering, Springer Berlin Heidelberg, pp :125-148, DOI:10.1007/978-3-642-15187-3_8.

[13] Pressman, R., (2010), "Software Engineering: A Practitioner's Approach", 7th Edition, McGraw-Hill, New York, USA, ISBN 978- 
0-07-337597-7.

[14] Sommerville, I., (2001), "Software documentation", Literate progamming. Lancaster University, UK, Retrieved from www.literateprogramming.com/documentation.pdf. 